\title{
IMMERSION IN THE METASTABLE RANGE AND 2-LOCALIZATION
}

\author{
HENRY H. GLOVER AND GUIDO MISLIN ${ }^{1}$
}

\begin{abstract}
Our purpose is to study immersion properties in the metastable range using the techniques of localization of homotopy types. The main theorem states that immersion of a manifold $M$ in euclidean space in the metastable range depends only upon the homotopy type $M_{2}$, the localization of $M$ at the prime 2 .
\end{abstract}

Introduction. It follows from [2] that in the metastable range immersion of a closed manifold depends only upon its homotopy type. One has

THEOREM 0.1. Let $M$ and $N$ be homotopy equivalent closed differentiable manifolds of dimension $n$. Suppose $M$ immerses in $\boldsymbol{R}^{n+k}$ for some $k \geqq$ $[n / 2]+2$. Then so does $N$.

Namely if $f: M \rightarrow R^{n+k}$ is an immersion with normal bundle $\nu$, then one can extend $f$ to an immersion of the total space $E(\nu)$ of $\nu, f: E(\nu) \rightarrow \boldsymbol{R}^{n+k}$. The zero section $s: M \rightarrow E(v)$ is an embedding. If $\theta: N \rightarrow M$ denotes a homotopy equivalence, then, by [2], $s \theta$ is homotopic to an embedding since $\operatorname{dim} E(v)-\operatorname{dim} N=k \geqq[n / 2]+2$; denote such an embedding by $\bar{\theta}: N \rightarrow E(v)$. Then $\bar{f} \bar{\theta}$ immerses $N$ into $\boldsymbol{R}^{n+k}$.

Our purpose is to study the immersion properties in the metastable range using the technique of localization of homotopy types ([3], [8]), and to prove a stronger form of Theorem 0.1 involving only the homotopy type of $M$ at the prime 2, denoted by $M_{2}$. In order to be able to localize $M$ at 2 we will suppose that its homotopy type is simple, meaning that $M$ is connected and that $\pi_{1} M$ operates trivially on $\pi_{\#} M$. We prove

THEOREM 0.2. Let $M$ and $N$ be connected, simple, orientable and closed differentiable manifolds of dimension $n$ whose 2-localizations $M_{2}$ and $N_{2}$ are homotopy equivalent. Suppose $M$ immerses in $\boldsymbol{R}^{n+k}$ for some $k \geqq[n / 2]+1$. Then $N$ immerses in $\boldsymbol{R}^{n+2[k / 2]+1}$.

Corollary 0.3. Let $M$ and $N$ be as in 0.2 and assume that $N$ is a $\pi$-manifold. Then $M$ immerses in $\boldsymbol{R}^{n+2[(n+2) / 4]+1}$.

\footnotetext{
Received by the editors June 5, 1973.

AMS (MOS) subject classifications (1970). Primary 57D40.

Key words and phrases. Immersion, localization.

1 Partially supported by NSF Grant GP-29544X.
} 
In particular this gives the following immersion theorem for generalized spherical space forms (compare Theorem $\mathrm{C}$ of [6] in the case of Lens spaces).

COROLlaRY 0.4. Let $M=\Sigma^{2 n+1} / G$, where $G$ is a finite abelian group of odd order operating fixed point free and smoothly on the homotopy sphere $\Sigma^{2 n+1}$. Then $M$ immerses in $R^{2[3(n+1) / 2]}$.

1. Localization. We will use the notation and results of [3] and [8]. If $X$ is a connected simple CW homotopy type then $X_{p}$ denotes its $p$ localization ( $p$ a prime or 0 ); there are canonical maps $X \rightarrow X_{p}$ respectively $X_{p} \rightarrow X_{0}$. We will need the following basic result of [3].

THEOREM 1.1. Let $W$ be a connected finite $\mathrm{CW}$ complex and $X$ a connected simple $\mathrm{CW}$ complex of finite type. Then the set of pointed homotopy classes $[W, X]$ is the pullback of the diagram of sets

$$
\left\{\left[W, X_{p}\right] \rightarrow\left[W, X_{0}\right] \mid p \in P\right\},
$$

$P$ denoting the set of primes.

We will use this theorem in a situation where $X$ is simply connected. In this case we do not have to distinguish between free and pointed homotopy classes of maps.

2. Some facts about $S O$ and $S F$. In this section we reformulate some known results about the special orthogonal groups and $S F(q)$, the monoid of degree one pointed maps of $S^{q}$. By suspension there is an inclusion $S F(q) \subset S F(q+1)$. Let $S F=\bigcup S F(q)$. The canonical maps $S O(q) \rightarrow S F(q)$ and $S O \rightarrow S F$ induce a map of pairs

$$
\theta:(S O, S O(q)) \rightarrow(S F, S F(q)) .
$$

By $[5,3.2]$ one has

LEMMA 2.1. The canonical map $\theta_{\#}: \pi_{n}(S O, S O(q)) \rightarrow \pi_{n}(S F, S F(q))$ is an isomorphism if $n \leqq 2 q-2$.

In accordance with $\S 1$ we write $S O_{p}$ for the $p$-localization of $S O$. The reader should not confuse $S O_{p}$ with $S O(p)$.

LEMMA 2.2. Let $q$ be an odd integer and $p$ an odd prime. Then

(i) $\pi_{k}\left(S O_{p}, S O(q)_{p}\right)=0$ for $k \leqq 2 q$;

(ii) $\pi_{k}\left(S F_{p}, S F(q)_{p}\right)=0$ for $k \leqq(p-1)(q+1)-2$.

Proof. The first result follows by the relative Hurewicz theorem from $H_{k}\left(S O_{p}, S O(q)_{p}: Z\right)=0$ for $k \leqq 2 q$. The second result follows from the isomorphisms $\pi_{k} S F(q) \cong \pi_{k+q} S^{q}$ and $\pi_{k} S F \cong \pi_{k}^{s t} S^{0}$ and the fact that 
$\left(\pi_{k+q} S^{q}\right)_{p} \rightarrow\left(\pi_{k}^{s t} S^{0}\right)_{p}$ is an isomorphism ( $p$ and $q$ odd) if $k<(p-1)(q+1)-2$ and surjective for $k=(p-1)(q+1)-2$.

We will use the following lemma on liftings in fiber spaces which is proved in $[9,3.2]$.

LEMMA 2.3. Consider the following diagram of connected $\mathrm{CW}$ complexes in which the columns are fibrations:

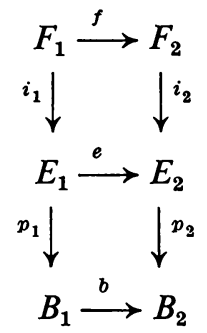

Suppose $f_{\#}: \pi_{k} F_{1} \rightarrow \pi_{k} F_{2}$ is an isomorphism for $k<n$ and suppose given a $\mathrm{CW}$ complex $X$ of dimension $n$ together with maps $g: X \rightarrow B_{1}, h: X \rightarrow E_{2}$ such that $b g=p_{2} h$. Then there exists a map $\tilde{g}: X \rightarrow E_{1}$ with $p_{1} \tilde{g}=g$.

3. Geometric dimension. Let $X$ be a connected finite CW complex. If $\alpha \in[X, B S O]=\widetilde{K} O(X)$ is an oriented stable bundle over $X$, then we denote by $J \alpha, \alpha_{p}$, respectively $J \alpha_{p}$, the canonical image of $\alpha$ in $[X, B S F]$, $\left[X, B S O_{p}\right]$, respectively $\left[X, B S F_{p}\right]$. As usual, we define the geometric dimension of $\alpha \in[X, B S O]$ by

$$
\operatorname{gd}(\alpha)=\min \{j \mid \alpha \in \operatorname{im}([X, B S O(j)] \rightarrow[X, B S O])\} .
$$

Since $\pi_{i}(S O, S O(j))=0$ for $i<j$, we see that $\operatorname{gd}(\alpha) \leqq \operatorname{dim} X$. We also define, for $\beta \in[X, B S F]$,

$$
\operatorname{gd}(\beta)=\min \{j \mid \beta \in \operatorname{im}([X, B S F(j)] \rightarrow[X, B S F])\} .
$$

Again $\pi_{i}(S F, S F(j))=0$ for $i<j$, so $\operatorname{gd}(\beta) \leqq \operatorname{dim} X$. Similarly we define, for $\gamma \in\left[X, B S O_{p}\right]$, respectively $\delta \in\left[X, B S G_{p}\right]$,

$$
\begin{aligned}
& \operatorname{gd}(\gamma)=\min \left\{j \mid \gamma \in \operatorname{im}\left(\left[X, B S O(j)_{p}\right] \rightarrow\left[X, B S O_{p}\right]\right)\right\}, \\
& \operatorname{gd}(\delta)=\min \left\{j \mid \delta \in \operatorname{im}\left(\left[X, B S F(j)_{p}\right] \rightarrow\left[X, B S F_{p}\right]\right)\right\} .
\end{aligned}
$$

In the following, $X$ will always denote a finite connected CW complex.

Lemma 3.1. Let $\alpha \in[X, B S O]$ and $\operatorname{dim} X=n$. Then for $p$ odd, $\operatorname{gd}\left(\alpha_{p}\right) \leqq$ $2[n / 4]+1$.

Proof. By Lemma 2.2, $\left(S O_{p}, S O(2[n / 4]+1)_{p}\right)$ is $4[n / 4]+2$ connected. Since $4[n / 4]+2 \geqq n-1$ we see that $\tilde{H}^{i}\left(X ; \pi_{i-1}\left(S O_{p}, S O(2[n / 4]+1)_{p}\right)\right)=0$ for all $i$ and hence every map $\alpha_{p}: X \rightarrow B S O_{p}$ lifts to $B S O(2[n / 4]+1)_{p}$. Hence the result. 
Recall that $n$ is any positive integer and $p$ is any odd prime. We next define an integer $\varepsilon(n, p)$ by the equation

$$
[n /(p-1)]+\varepsilon(n, p)=\min \{2 j+1 \mid 2 j+1 \geqq((n+1) /(p-1))-1\} \text {. }
$$

Notice that $\varepsilon(n, p)=0,1$ for all such $n$ and $p$.

Lemma 3.2. Let $\beta \in[X, B S F], \operatorname{dim} X=n$, and $p$ be any odd prime. Then $\operatorname{gd}\left(\beta_{p}\right) \leqq[n /(p-1)]+\varepsilon(n, p)$.

Proof. $\left(S F_{p}, S F([n /(p-1)]+\varepsilon(n, p))_{p}\right)$ is

$$
(p-1)([n /(p-1)]+\varepsilon(n, p)+1)-2
$$

connected by Lemma 2.2. Since

$$
(p-1)([n /(p-1)]+\varepsilon(n, p)+1)-2 \geqq n-1
$$

we see that

$$
\tilde{H}^{i}\left(X ; \pi_{i-1}\left(S F_{p}, S F([n / p]+\varepsilon(n, p))_{p}\right)\right)=0 \text { for all } i .
$$

Hence the result.

LEMma 3.3. Let $\alpha \in[X, B S O], \operatorname{dim} X=n$, and suppose there exists $a$ $k>[n / 2]$ such that $\operatorname{gd}(J \alpha) \leqq k$. Then $\operatorname{gd}(\alpha) \leqq k$.

Proof. Since $\operatorname{gd}(J \alpha) \leqq k$ it follows by Lemma 2.3 that we can lift $\alpha: X \rightarrow B S O$ into $B S O(k)$ provided that can $: \pi_{i}(S O, S O(k)) \rightarrow \pi_{i}(S F, S F(k))$ is an isomorphism for $i<n$. But this is the case by Lemma 2.1, since $k>[n / 2]$ and therefore $2 k-2 \geqq n-1$.

LEMma 3.4. Let $\beta \in[X, B S F]$. If $\max \left\{\operatorname{gd}\left(\beta_{p}\right) \mid p \in P\right\} \leqq 2 k+1$, then $\operatorname{gd}(\beta) \leqq 2 k+1$.

Proof. Notice that

$$
B S F(2 k+1) \cong \prod_{p \in P} B S F(2 k+1)_{p}
$$

since $\pi_{i} B S F(2 k+1)$ is finite for all $i$. Hence the result.

Proposition 3.5. Let $\alpha \in[X, B S O], \operatorname{dim} X=n, k>[n / 2]$ and $\operatorname{gd}\left(J \alpha_{2}\right) \leqq$ $k$. Then $\operatorname{gd}(\alpha) \leqq 2[k / 2]+1$.

Proof. By hypothesis $\operatorname{gd}\left(J \alpha_{2}\right) \leqq k \leqq 2[k / 2]+1$ and, by Lemma 3.2, $\operatorname{gd}\left(J \alpha_{p}\right) \leqq[n / 2]+\varepsilon(n, 3) \leqq 2[k / 2]+1$ for all odd primes $p$. Hence 3.4 implies that $\operatorname{gd}(J \alpha) \leqq 2[k / 2]+1$. Since $2[k / 2]+1 \geqq k>[n / 2]$ we conclude by 3.3 that $\operatorname{gd}(\alpha) \leqq 2[k / 2]+1$.

4. The proof of Theorem 0.2 and corollaries. For a connected closed differentiable manifold $M$ denote by $\tau(M) \in[M, B O]=\tilde{K} O(M)$ the 
stable tangent bundle and by $v(M)=-\tau(M)$ the stable normal bundle. The following proposition is well known in the corresponding "global" situation $[1,3.6]$.

Proposition 4.1. Let $M$ and $N$ be connected, simple and closed differentiable manifolds and $\varphi: N_{2} \rightarrow M_{2}$ a homotopy equivalence. Then

$$
\varphi * J v(M)_{2}=J v(N)_{2}
$$

in $\left[N_{2}, B F_{2}\right] \cong\left[N, B F_{2}\right]$.

Proof. Suppose $\varphi * J v(M)_{2}=w \neq J v(N)_{2}$. Let $J v(N) \in[N, B F]$ be the stable normal fibration. Since $B F \cong \prod_{p \in P} B F_{p}$ we can define an element $\theta \in[N, B F]$ by giving $\theta_{p} \in J v(N)_{p}$ for $p$ odd and $\theta_{2}=w$. Note that $\theta=$ $J \boldsymbol{v}(N)$. We want this to lead to a contradiction by showing that the Thom complex $N^{\theta}$ is $S$-reducible. For $\alpha \in[N, B F]$ we define $\left(N_{p}\right)^{\alpha_{p}}$ in the obvious way: represent $\alpha_{p}$ by the $S_{p}^{k}$-fibration $p r: E_{p} \rightarrow N_{p}, k \geqq \operatorname{dim} M=\operatorname{dim} N$; then $\left(N_{p}\right)^{\alpha_{p}}$ is the $S$-type of the mapping cone of $p r$. There is a canonical $S$-map $\left(N^{\alpha}\right)_{p} \rightarrow\left(N_{p}\right)^{\alpha_{p}}$ which is an $S$-equivalence since it induces an isomorphism in homology. We call $N^{\alpha} S$-reducible at $p$ if the ( $p$-local) top cell of $\left(N_{p}\right)^{\alpha}$ splits off stably. Clearly $N^{\alpha}$ is $S$-reducible if and only if it is $S$-reducible at $p$ for all primes $p$ (apply Theorem 1.1). Since $\theta_{p}=J v(N)_{p}$ for $p$ odd we see that $N^{\theta}$ is $S$-reducible at all odd primes. Further $\left(N_{2}\right)^{\theta_{2}}=$ $\left(N_{2}\right)^{\theta} \cong\left(M_{2}\right)^{J v(M)_{2}}$ and hence $N^{\theta}$ is $S$-reducible at 2 . We conclude that $N^{\theta}$ is $S$-reducible. But by a theorem of Spivak [7] this implies that $\theta=J v(N)$ contradicting our assumption.

We can now prove our theorem. Let $M$ and $N$ be as stated in Theorem 0.2 . Denote by $\tilde{\tau}(M)$ the unique lift of $\tau(M): M \rightarrow B O \cong B S O \times \boldsymbol{R P}^{\infty}$ to $B S O$, the universal cover of $B O$, and let $\tilde{v}(M)=-\tilde{\tau}(M) \in \tilde{K} S O(M)$ be the oriented stable normal bundle. Since $M$ immerses in $\boldsymbol{R}^{n+k}$ we have $\operatorname{gd}\left(J \tilde{v}(M)_{2}\right) \leqq \operatorname{gd}(\tilde{v}(M)) \leqq k$. Let $\varphi: N_{2} \rightarrow M_{2}$ be a homotopy equivalence. By Proposition 4.1, $\varphi * J v(M)_{2}=J v(N)_{2}$ and hence $k \varphi * J \tilde{v}(M)_{2}=k J \tilde{v}(N)_{2}$ for $k: B S F_{2} \rightarrow B F_{2}$ the canonical map. Since $k$ is a 2 -fold covering up to homotopy, with covering transformations homotopic to the identity, every homotopy class into $B F_{2}$ which lifts to $B S F_{2}$ lifts in a unique way. Hence we have $\varphi * J \tilde{v}(M)_{2}=J \tilde{v}(N)_{2}$. We conclude that $\operatorname{gd}\left(J \tilde{v}(N)_{2}\right) \leqq$ $\operatorname{gd}\left(J \tilde{v}(M)_{2}\right) \leqq k$, and, by Proposition 3.5, $\operatorname{gd}(\tilde{v}(N)) \leqq 2[k / 2]+1$. By the theorem of Hirsch [4] this implies that $N$ immerses with codimension $2[k / 2]+1$.

The Corollary 0.3 follows immediately by observing that $N$ immerses with codimension 1 , in case $N$ is a $\pi$-manifold. We can therefore apply the theorem with $k=[n / 2]+1$.

For Corollary 0.4 one uses that the covering projection $\Sigma^{2 n+1} \rightarrow M$ induces an equivalence $\Sigma_{2}^{2 n+1} \cong M_{2}$, since $G$ is of odd order; notice that $M$ 
is simple since the operations of $G$ are all homotopic to the identity. The result now follows from Corollary 0.3 by observing that $\Sigma^{2 n+1}$ is a $\pi$ manifold.

\section{REFERENCES}

1. M. F. Atiyah, Thom complexes, Proc. London Math. Soc. (3) 11 (1961), 291-310. MR 24 \#A1727.

2. A. Haefliger, Plongements différentiable de variétés dans variétés, Comment. Math. Helv. 36 (1961), 47-82. MR 26 \#3069.

3. P. J. Hilton, G. Mislin and J. Roitberg, Homotopical localization, Proc. London Math. Soc. 26 (1973), 693-706.

4. M. W. Hirsch, Immersion of manifolds, Trans. Amer. Math. Soc. 93 (1959), 242276. MR 22 \#9980.

5. I. M. James, On the iterated suspension, Quart. J. Math. Oxford Ser. (2) 5 (1954), 1-10. MR 15, 891.

6. D. Sjerve, Geometric dimension of vector bundles over Lens spaces, Trans. Amer. Math. Soc. 134 (1968), 545-557. MR 38 \#1695.

7. M. Spivak, Spaces satisfying Poincaré duality, Topology 6 (1967), 77-101. MR 35 \#4923.

8. D. Sullivan, Geometric topology. Part I: Localization, periodicity, and Galois symmetry, MIT, June 1970, (mimeographed notes).

9. W. A. Sutherland, Fiber homotopy equivalence and vector fields, Proc. London Math. Soc. (3) 15 (1965), 543-556. MR 31 \#763.

ForschungSinstituT FÜR MATHEMATIK, ETH, ZÜRICH, SWITZERLAND

Current address: Department of Mathematics, Ohio State University, Columbus, Ohio 43210 